Revisión

\title{
Disbiosis como factor determinante de enfermedad oral y sistémica: importancia del microbioma
}

\author{
Eduardo Chimenos-Küstner ${ }^{\mathrm{a}, *}$, María Laura Giovannoni ${ }^{\mathrm{b}}$ y Mayra Schemel-Suárez ${ }^{\mathrm{c}}$

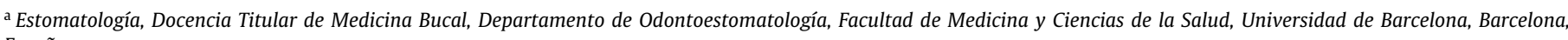 \\ España

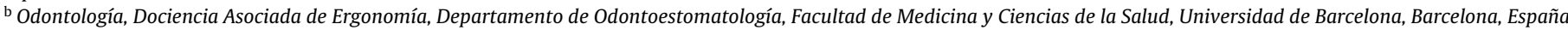 \\ c Odontología, Máster en Medicina Bucal, Departamento de Odontoestomatología, Facultad de Medicina y Ciencias de la Salud, Universidad de Barcelona, Barcelona, España
}

\section{INFORMACIÓN DEL ARTÍCULO}

\section{Historia del artículo:}

Recibido el 9 de mayo de 2017

Aceptado el 20 de mayo de 2017

On-line el xxx

\section{Palabras clave:}

Disbiosis

Microbiota

Microbioma

Medicina oral

Medicina periodontal

\section{R E S U M E N}

Los avances en los estudios de genética y epigenética modifican algunos conceptos de salud y enfermedad que se habían mantenido intactos durante décadas. En este sentido, en los últimos años se está otorgando un protagonismo creciente a microorganismos que han acompañado durante millones de años de evolución a los seres vivos superiores. Los genes de estos y de su microbiota constituyen un microbioma que interviene en el mantenimiento de la salud. La boca es lugar de asiento de gran variedad de microorganismos, cuyo control ayuda a estabilizar la enfermedad oral y sistémica. El objetivo del presente artículo es actualizar algunos conceptos relativos al microbioma oral y de su vinculación con la salud oral y general. (C) 2017 Elsevier España, S.L.U. Todos los derechos reservados.

\section{Dysbiosis as a determinant factor of systemic and oral pathology: importance of micorbiome}

\begin{abstract}
A B S T R A C T
Advances in genetic and epigenetic studies modified some concepts of health and disease that had been kept intact for decades. In this respect, in the last few years, microorganisms that have evolved with superior life forms for millions of years have taken an increased prominence. The genes of organisms and their microbiota constitute a microbiome that intervenes in health maintenance. The oral cavity is inhabited by a variety of microorganisms, their control aids in stabilising oral and systemic disease. The objective of this article is to update some concepts related to oral microbiome and its correlation with general and oral health.
\end{abstract}

(c) 2017 Elsevier España, S.L.U. All rights reserved.

\section{Introducción}

Algunos paradigmas sobre salud y enfermedad están cambiando en relación con la genética y la epigenética. Así, el estudio de la conjunción del genoma humano con el de la microbiota residente (microbioma) está en auge ${ }^{1}$. El análisis del microbioma ayuda a comprender la interacción entre la expresión y las funciones de

\footnotetext{
* Autor para correspondencia.

Correo electrónico: echimenos@ub.edu (E. Chimenos-Küstner).
}

nuestros propios genes y los de otros organismos, sobre todo bacterias. En este artículo se revisa el estado actual de conocimientos relativos al microbioma y se discuten implicaciones importantes en la salud oral y general.

Durante millones de años, nuestro microbioma ha coevolucionado con nosotros, desempeñando un papel significativo en nuestra fisiopatología. Existe evidencia de que los microbios residentes han estado desempeñando funciones metabólicas en los animales durante al menos 500 millones de años. El material genético de los microbios ha acompañado a los humanos en sus migraciones desde su lugar de nacimiento en África y se 
Tabla 1

Principales contribuciones funcionales atribuidas al microbioma humano

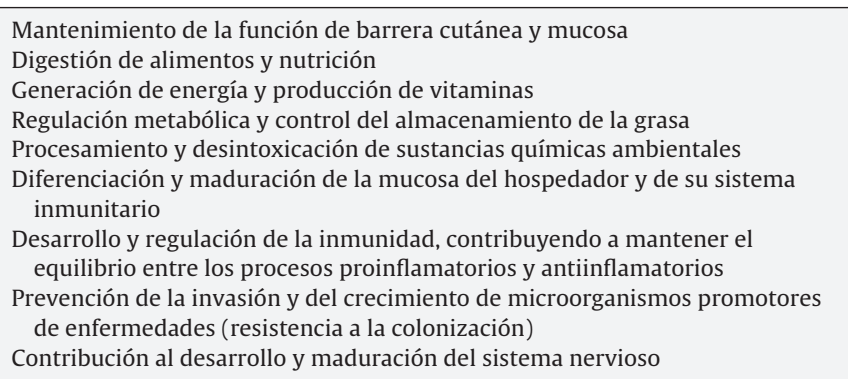

ha utilizado junto con marcadores humanos para rastrear rutas migratorias en todo el planeta. Como ejemplo, el seguimiento de cepas de Helicobacter pylori distingue poblaciones con mayor exactitud que marcadores genéticos humanos ${ }^{2,3}$.

Por otra parte, el conjunto de las relaciones y la organización de los tejidos durante el desarrollo conforma su constitución epigenética. Es un área clave de investigación, que combina aspectos de genética y medioambiente orientados hacia sistemas biológicos complejos $^{4}$. El medioambiente ha modelado continuamente la composición de nuestro microbioma, desde el Neolítico, durante la Revolución Industrial, hasta las épocas modernas. El descubrimiento del fuego, la invención de la agricultura, el creciente acceso a alimentos procesados, la exposición a metales pesados, biocidas, desinfectantes y antibióticos han contribuido a modificar la composición del microbioma humano ${ }^{2,5}$. La introducción de harinas y azúcares refinados en nuestra dieta ha favorecido que ciertas bacterias alteraran genéticamente su metabolismo. Así, Streptococcus mutans compite con éxito contra otras especies bacterianas orales, al aumentar sus defensas contra el estrés oxidativo y su resistencia ante metabolitos ácidos de los nuevos hidratos de carbono. Tras esta adaptación, aumenta su prevalencia en la cavidad oral, junto con la de otras especies tolerantes de ácido ${ }^{6}$.

Las comunidades microbianas humanas endógenas participan en múltiples funciones metabólicas, fisiológicas e inmunológicas (tabla 1), de ahí que la alteración de la función y de la composición del microbioma pueda tener consecuencias significativas para la salud $^{7,8}$

Nuestro microbioma se distribuye por los distintos compartimentos del cuerpo y es muy diverso y variable en y entre individuos. La mayor diversidad corresponde al tracto gastrointestinal y a la boca. La relación entre el microbioma y el hospedador es dinámica y está influida por muchos aspectos del estilo de vida moderno, que pueden desequilibrar el ecosistema. Distintos hábitats en la cavidad oral soportan comunidades microbianas heterogéneas, vinculadas con la salud oral y general. Para mantener un estado armónico de salud y prevenir la enfermedad, es necesario considerar al hospedador y sus residentes como un conjunto ${ }^{1,2,9}$.

\section{Particularidades del microbioma oral}

La boca alberga la segunda comunidad microbiana más diversa del cuerpo (después del intestino), con más de 700 especies de bacterias. Cuando se rompe el lábil equilibrio del ecosistema oral, se produce una disbiosis. Esta permite que las bacterias que promueven enfermedad se manifiesten y causen afecciones, tales como caries, gingivitis y periodontitis, que repercuten en la salud general $^{2}$. La colonización bacteriana se inicia en el nacimiento. La forma de adquisición de la microbiota (parto natural o cesárea) y la alimentación (lactancia natural o artificial) influyen en la diversidad posterior del microbioma oral y sistémico ${ }^{10,11}$. La lactancia materna, a los 3 meses de edad, ofrece una mayor colonización de lactobacilos orales que las leches artificiales. La erupción de los dientes proporciona nuevas superficies para la colonización microbiana y constituye un episodio ecológico importante en la boca infantil. El reemplazo de los dientes primarios por la dentición definitiva nuevamente modifica significativamente el hábitat microbiano oral ${ }^{2,12}$.

La colonización del medio oral está favorecida por la presencia de una fina capa mucilaginosa, denominada película adquirida. Dicha capa está constituida por proteínas, lípidos y otros componentes (hidratos de carbono, ácidos nucleicos), procedentes principalmente de la saliva, pero también derivadas del líquido crevicular (del surco gingival), de la mucosa oral y de las bacterias. Dicha película modula la unión de las bacterias a las superficies dentarias y epiteliales y protege la mucosa ${ }^{13}$. La composición individual de la película adquirida favorece la adherencia bacteriana, con la que interactúa. La película colonizada constituye la placa o biofilm, que conforma una superficie con actividad biológica. La saliva contribuye al mantenimiento y control del biofilm y modula las capas de placa bacteriana, con la participación de numerosas proteínas y minerales, como inmunoglobulina A secretora, lactoferrina, lactoperoxidasa, lisozima, estaterina e histatinas. Saliva y líquido crevicular proporcionan nutrientes para el crecimiento microbiano y contienen componentes con actividades antimicrobianas ${ }^{14,15}$.

Colaboración y antagonismo entre especies del biofilm contribuyen a la estabilidad ecológica, repercuten en la virulencia y el potencial patogénico de las bacterias e intervienen en la tolerancia a las defensas del hospedador y a los agentes antimicrobianos ${ }^{16}$. En la boca se reconocen varios hábitats distintos de colonización microbiana, tales como dientes, surco gingival, encía, lengua, mejilla, labios y paladar. Constituyen un sistema ecológico muy heterogéneo y favorecen el crecimiento de comunidades microbianas diferentes, sobre la película adquirida previa ${ }^{17}$. Las bacterias residentes tienen actividades pro y antiinflamatorias, cruciales para mantener la homeostasis en la cavidad oral ${ }^{18}$. Las infecciones agudas de la mucosa oral son bastante raras, a pesar de la densa colonización microbiana ${ }^{19}$. Cabe atribuirlo a las interacciones hospedador-microbio, cuya importancia se pone de relieve al observar a pacientes inmunodeprimidos, que pueden experimentar infecciones bacterianas, víricas y fúngicas potencialmente mortales ${ }^{2}$.

La promoción de medidas higiénicas a finales del siglo XIX en el mundo desarrollado (cepillado, uso de seda dental) ${ }^{20}$ ha contribuido a cambiar la composición del microbioma oral ${ }^{21}$. Asimismo, el consumo de bebidas azucaradas y azúcares refinados o el hábito tabáquico probablemente hayan tenido un impacto adicional en el ecosistema oral ${ }^{22}$, propiciando afecciones como caries y enfermedad periodontal y favoreciendo el predominio de determinados microorganismos en el biofilm, con repercusiones organolépticas desagradables, como la halitosis 23,24 .

\section{Repercusiones sistémicas}

La microbiota oral interviene también en procesos sistémicos. Sirva como ejemplo el efecto de las bacterias orales que expresan nitrato-reductasa, que catalizan la conversión de los nitratos de la dieta en nitritos. Al ser tragado, el nitrito salival se convierte en óxido nítrico, potente vasodilatador con actividad antimicrobiana, que desempeña un papel importante en el mantenimiento de la salud cardiovascular. El nitrito también estimula la producción de moco gástrico. Un consumo moderado de nitrato reduce la presión sanguínea, inhibe la función plaquetaria y mejora la función endotelial; la ingestión sostenida de nitratos en la dieta mejora la función vascular en pacientes con hipercolesterolemia. Estas mejoras se asocian con cambios en el microbioma oral en favor de organismos que son capaces de reducir los nitritos ${ }^{2,25}$. Sin embargo, mientras los nitratos de la dieta aumentan la formación de nitrito, el óxido 
nítrico en los tejidos interactúa con radicales superóxido liberados por las células inmunitarias, formando aniones peroxinitrito, con perjuicio celular. Al parecer, varios estudios a pequeña escala han demostrado que el uso de colutorios para enjuagues bucales que contienen clorhexidina puede reducir la concentración de nitritos tanto en la saliva como en el plasma, contribuyendo al control de la presión arterial. El tema de los efectos de nitrato/nitrito/óxido nítrico sobre la salud aún es controvertido, por lo cual estos interesantes datos requieren confirmación con estudios a mayor escala, que aporten conclusiones más firmes ${ }^{2,26,27}$.

La relación entre patógenos orales y efectos sistémicos se ha evidenciado en animales, donde la administración oral de Porphyromonas gingivalis cambió la composición del microbioma intestinal, provocando procesos inflamatorios en diversos tejidos y órganos ${ }^{28}$. Las bacterias orales desempeñan un papel en diversas afecciones sistémicas, incluyendo enfermedad cardiovascular, artritis reumatoide, efectos adversos en el embarazo, ictus, enfermedad intestinal inflamatoria y cáncer colorrectal, infecciones del aparato respiratorio, meningitis o abscesos cerebrales, abscesos en pulmón, hígado y bazo, apendicitis, neumonía y diabetes ${ }^{17,24,29-34}$. La periodontitis avanzada dificulta el control glucémico en la diabetes y en sujetos que no la padecen. Asimismo, la periodontitis avanzada se asocia a un mayor riesgo de diabetes tipo $2 \mathrm{y}$, en pacientes diabéticos, existe una correlación directa entre la gravedad de la periodontitis y las complicaciones de la enfermedad. En los últimos años se ha desarrollado un interés creciente por aclarar la posible relación de las enfermedades periodontales con determinados procesos sistémicos, como son episodios isquémicos cardiovasculares, partos prematuros y/o neonatos con bajo peso al nacer, obesidad, diabetes mellitus, infecciones pulmonares, etc. ${ }^{30}$. Todos estos procesos inflamatorios crónicos tienen un nexo de unión con la periodontitis, lo que ha motivado el interés actual por el concepto de medicina periodontal ${ }^{35}$. Este hecho es relevante, ya que la enfermedad crónica de declaración no obligatoria (enfermedades cardiovasculares, cáncer, enfermedades respiratorias crónicas y diabetes) supone la primera causa de muerte y discapacidad en el mundo y provoca más muertes actualmente que todas las demás enfermedades combinadas, en los países muy industrializados ${ }^{36}$.

La alteración del microbioma se ha vinculado a: 1) exposición a moléculas perturbadoras (ingredientes alimentarios como azúcares, gluten, agua clorada, antibióticos, multitud de productos químicos); 2) escasez de nutrientes que fomenten colonias saludables de bacterias (dietas deficientes en vegetales con fibra o con excesivas grasas saturadas), y 3) situaciones que provoquen y mantengan estrés. Como consecuencia, el microbioma alterado contribuye a un estado de inflamación crónica que predispone a enfermedades tan variadas como asma, alergias diversas, obesidad, diabetes, cáncer, depresión, autismo, artritis, cardiopatía isquémica, esclerosis múltiple, enfermedad de Parkinson y alzhéimer ${ }^{2}$. La alteración del microbioma y los procesos inflamatorios crónicos derivados favorecen la permeabilidad de la mucosa de los diferentes tractos afectados (ojos, nariz, boca, garganta, vías respiratorias, tracto gastrointestinal, vías urogenitales), que constituyen amplias vías de entrada para patógenos potenciales. Las actuales líneas de investigación, en relación con estos aspectos, están cambiando muchos conceptos diagnósticos y terapéuticos clásicos $2,37,38$.

\section{Discusión}

Cuando las especies de microorganismos que residen en la cavidad oral o en otras superficies corporales se mantienen en equilibrio (simbiosis), se habla de estado saludable. Por el contrario, la ruptura de dicho equilibrio (disbiosis) se asocia con enfermedad y se caracteriza por la alteración de la diversidad y las proporciones relativas de especies de la microbiota ${ }^{39}$. La relación entre el microbioma oral y su hospedador es dinámica. En la boca sana la composición de las comunidades microbianas es muy estable, pero los cambios biológicos en la vida de una persona pueden influir en el equilibrio de las especies dentro de estas comunidades ${ }^{40}$. A los cambios fisiológicos propios de la edad o cambios hormonales de la pubertad y el embarazo, los individuos sanos suelen adaptarse sin perjuicio de su salud bucal ${ }^{41}$. En otras situaciones, la alteración del ecosistema bucal puede causar disbiosis y pérdida del equilibrio o diversidad de la comunidad del biofilm, predominando una sola o pocas especies y aumentando el riesgo asociado de enfermedad ${ }^{42}$. Son factores predisponentes a disbiosis oral la disfunción de las glándulas salivales (cambios en el flujo y/o la composición de la saliva), mala higiene bucal, inflamación gingival y estilos de vida, incluida la alimentación y los hábitos tóxicos (como consumo de tabaco fumado o mascado, solo o asociado a otras sustancias), así como determinadas conductas sexuales ${ }^{11,43-46}$. Actualmente se considera que las bacterias etiquetadas como patógenos orales se pueden encontrar en un número reducido en sitios saludables y que la enfermedad oral ocurre como consecuencia de un cambio deletéreo en el equilibrio natural de la microbiota, más que como resultado de una "infección» exógena ${ }^{40}$. La disbiosis de la enfermedad periodontal, como causa de bacteriemia, probablemente favorezca la diseminación sistémica de bacterias orales. Por tanto, será preceptiva una buena higiene bucal para controlar la carga bacteriana total $^{24}$.

Como forma de combatir la disbiosis bacteriana, numerosos autores proponen la administración de probióticos (contienen cepas de bacterias y/o levaduras beneficiosas) y prebióticos (alimentos ricos en fibra) para regular el equilibrio del microbioma. En algunos países se promueven asimismo trasplantes fecales (de comunidades bacterianas saludables), al parecer con éxito creciente en el tratamiento y la prevención de diversas enfermedades sistémicas $^{47-49}$.

Estos tratamientos no invasivos pueden compensar el uso indiscriminado de antibióticos, pero la variedad y el número de especies a reimplantar de esta forma son limitados y pueden no ser sostenibles. Por esta razón, deben acompañarse de una alimentación equilibrada y de la ingesta de prebióticos ${ }^{50}$. La dieta mediterránea parece ser un buen modelo a promover. Por otra parte, alimentos ecológicos (de origen animal o vegetal), menos procesados y de menor manipulación en su producción, ofrecen mayores garantías de respetar el microbioma que los manipulados genéticamente o con productos químicos (agroquímicos, antibióticos, hormonas, etc.). Por otra parte, es prioritario el aporte de líquidos. Fundamental para la supervivencia, el agua es vehículo de diversos elementos y sales minerales. La cloración de las aguas puede influir negativamente en la homeostasis del microbioma, por lo que debería ser un factor a considerar ${ }^{51}$. En definitiva, para prevenir y combatir la disbiosis se recomiendan medidas como las resumidas en la tabla 2.

\section{Tabla 2}

Medidas recomendables para reducir procesos disbióticos

Higiene oral regular (para mantener un control de la microbiota de la boca) Dieta saludable (con abundantes hortalizas y vegetales y escasos alimentos con harinas y azúcares refinados)

Mantenerse en un IMC adecuado (para evitar el síndrome metabólico), controlando el peso, la presión arterial y haciendo ejercicio aeróbico de forma regular

Evitar grasas hidrogenadas y saturadas, priorizando los ácidos grasos monoinsaturados (aceite de oliva) y poliinsaturados omega-3 (más que omega-6)

Evitar la administración indiscriminada e injustificada de antibióticos, así como la ingesta de carne de animales que hayan sido tratados con antibióticos o con hormonas

Restringir en lo posible los productos de cosmética o los alimentos que contengan moléculas de dudosa eficacia o de toxicidad reconocida (disruptores endocrinos) 
Si bien en párrafos anteriores se ha comentado la interacción de la microbiota bacteriana con el genoma humano, el proceso coevolutivo del microbioma humano ha implicado otros microorganismos, portadores de su respectiva carga genética, como hongos unicelulares (levaduras), protozoos y virus. Todos ellos han ido incorporándose y evolucionando, formando parte de nichos ecológicos (piel, boca, sistema digestivo, sistema respiratorio, aparato genital) y manteniendo entre sí una relación de equilibrio. Los avances tecnológicos y de toda índole que se han producido en el último siglo, sobre todo en el mundo occidental (en relación con estilos de vida, alimentación, higiene, hábitos tóxicos, fármacos, pesticidas, etc.), han propiciado un cambio esencial del microbioma, sin tiempo a una adaptación. De ahí los mayores índices de determinadas formas de enfermedad, más prevalentes en el mundo llamado civilizado.

Un papel esencial cabe atribuirlo a los virus. Aun sin considerarse seres vivos, son capaces de actuar sobre el genoma de forma muy patente ${ }^{52,53}$. Son nanopartículas que se comportan como parásitos intracelulares. Parasitan todo tipo de células, incluidas las bacterias (bacteriófagos), induciendo la formación y la diseminación de su información genética por distintos mecanismos y vienen haciéndolo desde el inicio de la vida en el planeta (quizá con papel protagonista en el proceso evolutivo) ${ }^{54}$. Tal vez determinadas enfermedades, como la considerada autoinmunitaria, obedezca a procesos disbióticos en los que partículas como los virus actúen modificando algunas proteínas. Su acción podría justificar patrones reactivos tegumentarios, como las manifestaciones liquenoides o penfigoides, o de afección ulcerosa sistémica, aftosa o aftoide, como en las enfermedades de Crohn, la colitis ulcerosa o la enfermedad de Behçet. Cabe cuestionarse si dichas entidades nosológicas son trastornos en sí mismos o son más bien manifestaciones de carácter inflamatorio, con participación de un microbioma alterado ${ }^{55-57}$. La acción de los virus sobre el material genético (ARN, ADN) puede modificar la expresión de protooncogenes y oncogenes, facilitando el desarrollo de cáncer ${ }^{58,59}$. El empleo de partículas tipo virus en nanomedicina ayuda a la detección, la prevención (vacunas), el diagnóstico y la mejora del tratamiento de enfermedades como el cáncer o la dolencia cardiovascular. Con toda probabilidad, esta será también una línea a potenciar en un futuro próximo ${ }^{60}$.

Para concluir, es evidente que el microbioma oral y sistémico están íntimamente relacionados y que su alteración (disbiosis) tiene grandes repercusiones en la salud. Por tanto, el diagnóstico y tratamiento de la afección oral contribuye al éxito terapéutico y a la prevención de enfermedad sistémica, que debe ser entendida de forma holística.

\section{Conflicto de intereses}

Los autores declaran no tener ningún conflicto de intereses.

\section{Bibliografía}

1. Methé BA, Nelson KE, Pop M, Creasy HH, Giglio MG, Huttenhower C, et al. A framework for human microbiome research. Nature. 2012;486:215-21.

2. Kilian M, Chapple ILC, Hannig M, Marsh PD, Meuric V, Pedersen AM, et al. The oral microbiome-An update for oral healthcare professionals. Br Dent J. 2016;221:657-66

3. Rinaldi A. Tiny travel companions. As microorganisms have accompanied mankind's journeys around the globe, they could help scientists to unravel our past. EMBO Rep. 2007;8:121-5.

4. Zerón A. Genoma, microbioma y epigenoma humano. Una visión contemporánea de la tríada ecológica. Rev ADM. 2014;71:162-70.

5. Gillings M, Paulsen I, Tetu S. Ecology and evolution of the human microbiota: Fire, farming and antibiotics. Genes (Basel). 2015;6:841-57

6. Cornejo OE, Lefébure T, Bitar PD, Lang P, Richards VP, Eilertson K, et al. Evolutionary and population genomics of the cavity causing bacteria Streptococcus mutans. Mol Biol Evol. 2013;30:881-93.

7. Krajmalnik-Brown R, Ilhan ZE, Kang DW, DiBaise JK. Effects of gut microbes on nutrient absorption and energy regulation. Nutr Clin Pract. 2012;27:201-14.
8. Relman DA. The human microbiome and the future practice of medicine. JAMA 2015;314:1127

9. Costello EK, Lauber CL, Hamady M, Fierer N, Gordon JI, Knight R. Bacterial community variation in human body habitats across space and time. Science. 2009;326:1694-7.

10. Lif Holgerson P, Harnevik L, Hernell O, Tanner AC, Johansson I. Mode of birth delivery affects oral microbiota in infants. J Dent Res. 2011;90:1183-8.

11. Jesús de la Calle I, Jesús de la Calle MA. Vaginosis bacteriana. Med Clin (Barc) 2009;133:789-97.

12. Holgerson PL, Vestman NR, Claesson R, Ohman C, Domellöf M, Tanner AC et al. Oral microbial profile discriminates breast-fed from formula-fed infants. J Pediatr Gastroenterol Nutr. 2013;56:127-36.

13. Hannig C, Hannig M, Attin T. Enzymes in the acquired enamel pellicle. Eur J Ora Sci. 2005;113:2-13.

14. Marsh PD, Do T, Beighton D, Devine DA. Influence of saliva on the oral microbiota. Periodontol 2000. 2016;70:80-92.

15. López-López J, Jané Salas E, Chimenos Küstner E. Pronóstico y tratamiento de la boca seca. Revisión sistemática. Med Clin (Barc). 2014:142:119-24.

16. Donlan RM, Costerton JW. Biofilms: Survival mechanisms of clinically relevant microorganisms. Clin Microbiol Rev. 2002;15:167-93.

17. Dewhirst FE, Chen T, Izard J, Paster BJ, Tanner AC, Yu WH, et al. The human oral microbiome. J Bacteriol. 2010;192:5002-17.

18. Devine DA, Marsh PD, Meade J. Modulation of host responses by oral commensal bacteria. J Oral Microbiol. 2015;7:26941.

19. Zaura E, Nicu EA, Krom BP, Keijser BJ. Acquiring and maintaining a normal oral microbiome: Current perspective. Front Cell Infect Microbiol. 2014;4:85.

20. American Dental Association. History of dentistry timeline [consultado 8 May 2017]. Disponible en: http://www.ada.org/en/about-the-ada/ada-historyand-presidents-of-the-ada/ada-history-of-dentistry-timeline

21. Marsh PD. Controlling the oral biofilm with antimicrobials. J Dent. 2010;38 Suppl $1:$ S11-5.

22. Adler CJ, Dobney K, Weyrich LS, Kaidonis J, Walker AW, Haak W, et al. Sequencing ancient calcified dental plaque shows changes in oral microbiota with dietary shifts of the Neolithic and Industrial revolutions. Nat Genet. 2013;45:450-5.

23. De Luca-Monasterios F, Chimenos-Küstner E, López-López J. Efecto de masticar chicle sobre la halitosis. Med Clin (Barc). 2014;143:64-7.

24. Han YW, Wang X. Mobile microbiome: Oral bacteria in extra-oral infections and inflammation. J Dent Res. 2013;92:485-91.

25. Velmurugan S, Gan JM, Rathod KS, Khambata RS, Ghosh SM, Hartley A et al. Dietary nitrate improves vascular function in patients with hypercholesterolemia: A randomized, double-blind, placebo-controlled study. Am J Clin Nutr. 2016:103:25-38.

26. Woessner M, Smoliga JM, Tarzia B, Stabler T, van Bruggen M, Allen JD. A stepwise reduction in plasma and salivary nitrite with increasing strengths of mouthwash following a dietary nitrate load. Nitric Oxide. 2016;54:1-7.

27. Kapil V, Haydar SM, Pearl V, Lundberg JO, Weitzberg E, Ahluwalia A. Physiological role for nitrate-reducing oral bacteria in blood pressure control. Free Radic Biol Med. 2013;55:93-100.

28. Arimatsu K, Yamada H, Miyazawa H, Minagawa T, Nakajima M, Ryder MI, et al. Oral pathobiont induces systemic inflammation and metabolic changes associated with alteration of gut microbiota. Sci Rep. 2014;4:4828.

29. Pedersen AML, editor. Oral infections and general health. Cham: Springer International Publishing Switzerland; 2016., http://dx.doi.org/10.1007/ 978-3-319-25091-5

30. Chapple IL, Genco R, working group 2 of the joint EFP/AAP workshop. Diabetes and periodontal diseases: Consensus report of the Joint EFP/AAP Workshop on Periodontitis and Systemic Diseases. J Periodontol. 2013;84 4 Suppl: S106-12.

31. De Pablo P, Chapple IL, Buckley CD, Dietrich T. Periodontitis in systemic rheumatic diseases. Nat Rev Rheumatol. 2009:5:218-24.

32. Rodríguez de Santiago E, Albillos Martínez A, López-Sanromán A. Infecciones en la enfermedad inflamatoria intestinal. Med Clin (Barc). 2017;148:415-23.

33. Sebastián Domingo JJ. Síndrome del intestino irritable: lo (pen)último sobre lo que se está investigando. Med Clin (Barc). 2016;146:260-2.

34. Tor J, Muga R. El hígado graso y el síndrome metabólico. Med Clin (Barc). 2013;141:252-3.

35. Williams RC, Offenbacher S. Periodontal medicine: The emergence of a new branch of periodontology. Periodontol 2000. 2000;23:9-12.

36. IARC Working Group on the Evaluation of Carcinogenic Risks to Humans. Betelquid and areca-nut chewing and some areca-nut derived nitrosamines. IARC Monogr Eval Carcinog Risks Humans. 2004:85:1-334.

37. Szot P, Franklin A, Figlewicz DP, Beuca TP, Bullock K, Hansen K, et al. Multiple lipopolysaccharide (LPS) injections alter interleukin 6 (IL-6), IL-7, IL-10 and IL-6 and IL-7 receptor mRNA in CNS and spleen. Neuroscience. 2017;355: $9-21$

38. Gomes C, Martinho FC, Barbosa DS, Antunes LS, Póvoa HC, Baltus TH, et al Increased root canal endotoxin levels are associated with chronic apical periodontitis, increased oxidative and nitrosative stress, major depression, severity of depression, and a lowered quality of life. Mol Neurobiol. 2017. doi: 10.1007/s12035-017-0545-z.

39. Cho I, Blaser MJ. The human microbiome: At the interface of health and disease. Nat Rev Genet. 2012;13:260-70.

40. Marsh PD, Head DA, Devine DA. Ecological approaches to oral biofilms: Contro without killing. Caries Res. 2015;49 Suppl 1:46-54.

41. Zaura E, Ten Cate JM. Towards understanding oral health. Caries Res. 2015;49 Suppl 1:55-61. 
42. Gross EL, Leys EJ, Gasparovich SR, Firestone ND, Schwartzbaum JA, Janies DA, et al. Bacterial 16S sequence analysis of severe caries in young permanent teeth. J Clin Microbiol. 2010;48:4121-8.

43. Wu J, Peters BA, Dominianni C, Zhang Y, Pei Z, Yang L, et al. Cigarette smoking and the oral microbiome in a large study of American adults. ISME J. 2016;10:2435-46.

44. Parmar G, Sangwan P, Vashi P, Kulkarni P, Kumar S. Effect of chewing a mixture of areca nut and tobacco on periodontal tissues and oral hygiene status. J Oral Sci. 2008;50:57-62.

45. Shrinkhala S, Roopa DA, Gopal S, Johari S, Pandey Y, Gupta T. Periodontal status among gutka chewers-A prevalence study. IJIRAS, 2016:3:284-9.

46. Javed F, Vohra F, Al-Kheraif AA, Malmstrom H, Romanos GE. Comparison of periodontal inflammatory conditions among habitual gutka chewers and betel quid chewers. Oral Dis. 2015;21:437-42.

47. Hu Y, Zhou F, Yuan Y, Xu Y. Efectos del suplemento de probióticos en pacientes con diabetes mellitus tipo 2: metaanálisis de ensayos aleatorizados. Med Clin (Barc). 2017;148:362-70.

48. Borody TJ, Paramsothy S, Agrawal G. Fecal microbiota transplantation: Indications, methods, evidence, and future directions. Curr Gastroenterol Rep. 2013;15:337.

49. Flannigan KL, Rajbar T, Moffat A, McKenzie LS, Dicke F, Rioux K, et al. Changes in composition of the gut bacterial microbiome after fecal microbiota transplantation for recurrent Clostridium difficile infection in a pediatric heart transplant patient. Front Cardiovasc Med. 2017;4:17

50. Di Bartolomeo F, Startek JB, van den Ende W. Prebiotics to fight diseases: Reality or fiction? Phytother Res. 2013;27:1457-73.
51. Revetta RP, Gomez-Alvarez V, Gerke TL, Santo Domingo JW, Ashbolt NJ. Changes in bacterial composition of biofilm in a metropolitan drinking water distribution system. J Appl Microbiol. 2016;121:294-305.

52. Puy D, Chimenos-Küstner E, Dorado C. Infección bucal por el virus del herpes simple: mecanismos patogénicos conocidos. Med Clin (Barc). 1998;111:105.

53. de Sousa Rodrigues PM, Teixeira AL, Chimenos Kustner E, Medeiros R. Are herpes virus associated to aggressive periodontitis? A review of literature. J Oral Maxillofac Pathol. 2015;19:348-55.

54. Ding ZF, Cao MJ, Zhu XS, Xu GH, Wang RL. Changes in the gut microbiome of the Chinese mitten crab (Eriocheir sinensis) in response to White spot syndrome virus (WSSV) infection. J Fish Dis. 2017. doi: 10.1111/jfd.12624.

55. Cervera Segura R, Font Franco J. Enfermedad autoinmune e infección: una relación bidireccional. Rev Clin Esp. 2003;203:167-9.

56. Schemel-Suárez M, López-López J, Chimenos-Küstner E. Oral ulcers: Differential diagnosis and treatment. Med Clin (Barc). 2015;145:499-503.

57. Chimenos-Küstner E, Larred-Conde N, Marques-Soares MS, López-López J. Manifestaciones orales de toxicidad sistémica: patología liquenoide. Piel. 2015;30:644-9.

58. Jané-Salas E, Chimenos-Küstner E, López-López J, Roselló-Llabrés X. Importance of diet in the prevention of oral cancer. Med Oral. 2003;8:260-8.

59. González Svatetz CA, Goday Arnó A. Obesidad y cáncer: «las amistades peligrosas». Med Clin (Barc). 2015;145:24-30.

60. Matoba T, Koga J, Nakano K, Egashira K, Tsutsui H. Nanoparticle-mediated drug delivery system for atherosclerotic cardiovascular disease. J Cardiol. 2017. pii: S0914-5087(17)30092-8. doi: 10.1016/j.jjcc.2017.03.005. 